\title{
Die Zukunft der
}

Betriebswirtschaftslehre

in Deutschland

Schriftleitung

Prof. Dr. Dr. h.c. Horst Albach

Prof. Dr. Klaus Brockhoff 
Editorial

Standortbestimmung der Betriebswirtschaftslehre

\section{Zukunft der Betriebswirtschaftslehre in Deutschland}

Thesen und Fragen zur "Zukunft der Betriebswirtschaftslehre in Deutschland"

Prof. Dr. Kurt Bohr, Regensburg

\section{Betriebswirtschaftslehre als Wissenschat}

Betriebswirtschaftslehre als Wissenschaft

Entwicklungstendenzen in der modernen Betriebswirtschaftslehre

Prof. Dr. Horst Albach, Bonn . . . . . . . . . . . . . . . . . . . . . . . . 7

\section{Lehre}

Plädoyer für eine bedürfnisgerechte Differenzierung der Ausbildung in der Betriebswirtschaftslehre

Prof. Dr. Klaus Brockhoff und Prof. Dr. Jürgen Hauschildt, Kiel . . . . . . . . . . . 27

Thesen zur Arbeitsteilung im Hochschulsystem

Prof. Dr. Dr. h.c. Otto H. Jacobs, Mannheim . . . . . . . . . . . . . . . . . . 41

Zur Standortbestimmung der Betriebswirtschaftslehre im Hochschulsystem

Prof. Dr. Marcell Schweitzer, Tübingen . . . . . . . . . . . . . . . . . . . . 49

Leistungsvergleiche mit dem Ausland

Die deutsche Betriebswirtschaftslehre im internationalen Wettbewerb

Prof. Dr. Hans Jürgen Drumm, Regensburg 
Die deutsche Betriebswirtschaftslehre im internationalen Wettbewerb ein Schwarzes Loch?

Prof. Dr. Hermann Simon, Mainz

Die deutsche betriebswirtschaftliche Forschung im internationalen Vergleich Prof. Dr. Klaus Macharzina, Stuttgart _ . . . . . . . . . . . . . . . . . . 85

Zur Effizienz betriebswirtschaftlicher Forschung im deutsch-amerikanischen Vergleich

Organisationsökonomische Analysen auf der Basis von Grenzproduktionsfunktionen Dr. Uschi Backes-Gellner, Trier

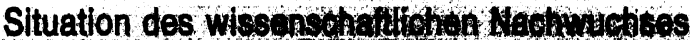

Die Entwicklung des Bestandes der Hochschullehrer für Betriebswirtschaft und mögliche Folgen

Prof. Dr. Dres. h.c. Eduard Gaugler, Mannheim

Die Habilitation als Voraussetzung für Hochschullehrer der Betriebswirtschaft

Prof. Dr. Arnold Picot, München

Wege aus der Not?

Versuche mit Volkswirten, Gastprofessoren und Graduiertenkollegs

Prof. Dr. Dieter Sadowski, Trier

Betriebswirtschaftslehre in den neven Bundeslăndern

Stellungnahmen und Empfehlungen des Wissenschaftsrates zu Forschung und Lehre in den neuen Bundesländern

Prof. Dr. Dieter Sadowski, Trier

Die Betriebswirtschaftslehre an den Universitäten der neuen Bundesländer

Prof. Dr. Dr. h.c. Karl Alewell, Gießen

Der Aufbau der Betriebswirtschaftslehre in Sachsen

Ein Zwischenbericht aus der Perspektive eines Gründungsdekans

Prof. Dr. Peter Rütger Wossidlo, Bayreuth

Fragen statt Antworten: Wie kann sich die Betriebswirtschaftslehre an ostdeutschen Fakultäten entwickeln?

Prof. Dr. Wulff Plinke, Berlin 


\section{Studiensituation in orof}

\section{Die Studiensituation in der Betriebswirtschaftslehre im Urteil der Studenten}

Priv.-Doz. Dr. Hans-Dieter Daniel, Mannheim, und Dipl.-Soz. Stefan Hornbostel, Jena

\section{Schlußwort}

Prof. Dr. Kurt Bohr, Regensburg

ZfB · Grundsätze und Ziele . . . . . . . . . . . . . . . . . . XVI

ZfB - Herausgeber . . . . . . . . . . . . . . . . . XVII

ZfB $\cdot$ Impressum $/$ Hinweise für Autoren . . . . . . . . . . . . . . . . . XVIII 


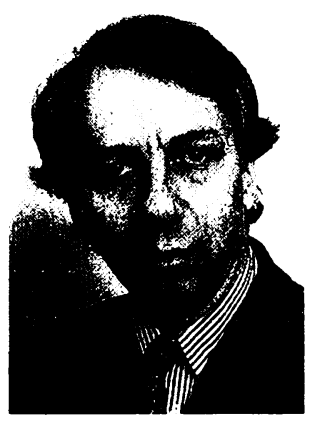

\title{
Die Habilitation als Voraussetzung für Hochschullehrer der Betriebswirtschaft
}

\author{
Von Arnold Picot
}

1. Für den Zugang zum Beruf des Hochschullehrers für Betriebswirtschaft ist die Habilitation eine wichtige Regelbedingung. Unter den Voraussetzungen der Bundesrepublik Deutschland sichert sie zusammen mit den anderen Qualifikationskriterien den Erhalt und die Weiterentwicklung der Universität als Stätte von wissenschaftlicher Forschung und Lehre.

2. Versuche, die mengenmäßige Entwicklung des betriebswirtschaftlichen Hochschullehrernachwuchses zentral zu beeinflussen, z. B. durch Empfehlungen des Verbandes oder durch Absprachen in und zwischen Fakultäten, sind untauglich und kontraproduktiv. Wegen der bekannten Probleme zentraler Prognosen und ihrer Fehlerwirkungen führen solche Bemühungen nicht nur nicht zu einem besseren Abgleich von Angebot und Nachfrage, sondern sie erzeugen in der Regel eine Übersteuerung (,Schweinezyklus“).

3. Die Entscheidung eines hochqualifizierten Nachwuchswissenschaftlers, den Berufsweg des Hochschullehrers einzuschlagen, ist dezentral sowie individuell zu treffen. Der potentielle Habilitand ist ein erwachsener Mensch, der über ausreichende Beratungsund Informationsmöglichkeiten verfügt, um Chancen und Risiken vor dem Hintergrund eigener Präferenzen abzuwägen. Eine feste Beschäftigungszusage kann einem Habilitanden nicht gegeben werden, aber auch nicht die Aussage, daß es keine Beschäftigung gibt.

4. Sofern eine Habilitation im unmittelbaren Anschluß an die Promotion angestrebt wird, sollte darauf geachtet werden, daß der Abschluß in möglichst jungem Alter erreicht wird (möglichst nicht älter als 35 Jahre), um die Verwendungsflexibilität nach der Habilitation in jeder Richtung (Praxis/Wissenschaft, wissenschaftliche Arbeitsgebiete usw.) zu erhalten.

5. Möglichkeiten, qualifizierte Praktiker für die Habilitation zu gewinnen, sollten vermehrt ausgeschöpft werden. Die zumeist finanziell wesentlich attraktiveren Vertragsbedingungen der Praxis beschränken allerdings diese Option. Dennoch gibt es positive Beispiele, die zeigen, daß ein solcher Weg vermehrt beschreitbar ist. Dazu ist u.a. auch

Professor Dr. Arnold Picot, Ludwig-Maximilians-Universität München, Ludwigstr. 28, 80539 München.

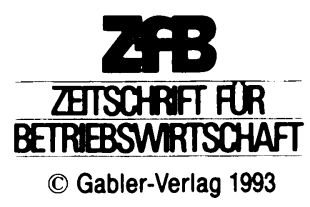




\section{Arnold Picot}

die Bereitschaft der Fakultäten zu verbessern, Externe als Habilitanden zu akzeptieren, sofern sie die erforderlichen Voraussetzungen erfüllen (Hochschul- und Lehrerfahrung, überdurchschnittliche Promotion, wissenschaftliche Publikationen). Es ist wünschenswert, daß Unternehmen solchen Nachwuchskräften geeignete Entfaltungsmöglichkeiten eröffnen, z. B. in Form von gemeinsamen Forschungsprojekten mit der Universität, Beurlaubungen, Freistellungen für Lehraufträge.

6. Die zunehmend zu beobachtenden Bemühungen von Unternehmen, die besten Absolventen bereits nach dem Diplom zu sehr guten finanziellen Bedingungen abzuwerben und ihnen zugleich, z. B. über ein ,,sabbatical“, die Promotion zu eröffnen, torpedieren die Notwendigkeit der Universität, den besten Absolventen Assistententätigkeit, Promotion und wissenschaftliche Perspektiven anzubieten. Dadurch werden dem Universitätsnachwuchs systematisch sehr gute Kandidaten entzogen. Die Universitäten sollten derartige Programme im Interesse der Sicherstellung der eigenen qualifizierten Forschung, Lehre und Nachwuchsentwicklung kritisch bewerten.

\section{Zusammenfassung}

Die Habilitation ist und bleibt die zentrale Qualifikationsvoraussetzung für den betriebswirtschaftlichen Hochschullehrernachwuchs an Universitäten. Es sind Bedingungen zu schaffen, damit die bestqualifizierten Universitätsabsolventen und auch Praktiker für Habilitation und Hochschullehrerberuf gewonnen werden können.

\section{Summary}

Habilitation continues to be a key-requirement for future professors in business administration on university level in Germany. Conditions must be improved in order to attract best qualified students and practitioners for an academic career in business administration. 